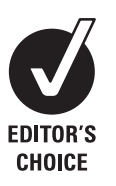

CHOICE

\title{
Systems ambiguity and guideline compliance: a qualitative study of how intensive care units follow evidence-based guidelines to reduce healthcare- associated infections
}

\author{
A P Gurses, ${ }^{1}$ K L Seidl, ${ }^{2}$ V Vaidya, ${ }^{3}$ G Bochicchio, ${ }^{4,5,6}$ A D Harris, ${ }^{2,7}$ J Hebden, ${ }^{8}$ Y Xiao ${ }^{5,9}$
}

${ }^{1}$ Division of Health Policy and Management, University of Minnesota, Minneapolis, Minnesota, USA; ${ }^{2}$ University of Maryland Medical Center, Baltimore, Maryland, USA; ${ }^{3}$ Department of Pediatrics, University of Maryland School of Medicine, Baltimore, Maryland, USA; ${ }^{4}$ Department of Surgery, University of Maryland School of Medicine, Baltimore, Maryland, USA; ${ }^{5}$ Program in Trauma, University of Maryland, Baltimore, Maryland, USA ${ }^{6}$ Veterans Affairs Medical Center, Baltimore, Maryland, USA; ${ }^{7}$ Department of Epidemiology and Preventive Medicine, University of Maryland, Baltimore, Maryland, USA; ${ }^{8}$ Infection Control and Hospital Epidemiology, University of Maryland Medical Center, Baltimore, Maryland, USA;

${ }^{9}$ Department of Anesthesiology, University of Maryland, Baltimore, Maryland, USA

\section{Correspondence to:}

A P Gurses, Division of Health

Policy and Management, University of Minnesota, 420

Delaware Street SE, Mayo Mail Code 729, Minneapolis, MN

55455,USA; gurse001@umn.edu

Accepted 19 September 2007

\section{ABSTRACT}

Background: Consistent compliance with evidencebased guidelines is challenging yet critical to patient safety. We conducted a qualitative study to explore the underlying causes for non-compliance with evidencebased guidelines aimed at preventing four types of healthcare-associated infections in the surgical intensive care unit (SICU) setting.

Methods: Twenty semistructured interviews were conducted with attending physicians (3), residents (2), nurses (6), quality improvement coordinators (3), infection control practitioners (2), respiratory therapists (2) and pharmacists (2) in two SICUs. Using a grounded theory approach, we performed thematic analyses of the interviews.

Results: The concept of systems ambiguity to explain noncompliance with evidence-based guidelines emerged from the data. Ambiguities hindering consistent compliance were related to tasks, responsibilities, methods, expectations and exceptions. Strategies reported to reduce ambiguity included clarification of expectations from care providers with respect to guideline compliance through education, use of visual cues to indicate the status of patients with respect to a particular guideline, development of tools that provide an overview of information critical for guideline compliance, use of standardised orders, clarification of roles of care providers and use of decision-support tools.

Conclusions: The concept of systems ambiguity is useful to understand causes of non-compliance with evidencebased guidelines aimed at reducing healthcare-associated infections. Multi-faceted interventions are needed to reduce different ambiguity types, hence to improve guideline compliance.

Consistent compliance with evidence-based guidelines can significantly improve patient safety and quality of care. ${ }^{12}$ Numerous quality improvement organisations are undertaking efforts to improve guideline compliance. For example, the Agency for Healthcare Research and Quality (AHRQ) has developed a comprehensive database of evidencebased guidelines and related documents (National Guideline Clearinghouse: http://www.guideline. gov) to facilitate implementation in the clinical setting. Recently, the Institute for Healthcare Improvement (IHI) enrolled over 3100 hospitals in a nationwide effort to save lives by endorsing implementation strategies aimed at facilitating compliance with guidelines. These and other efforts are aimed at reducing inconsistency in compliance with evidence-based guidelines, an area with significant room for improvement.
By definition, healthcare-associated infections (HAI) are potentially preventable harms. Reduction of these infections is a critical component of patient safety efforts. ${ }^{3}{ }^{4}$ Evidence-based guidelines have been developed to reduce HAI. The Healthcare Infection Control Practices Advisory Committee (HICPAC), a federal advisory committee, has developed several guidelines for the prevention of HAI including hand hygiene in healthcare settings, ${ }^{5}$ infection control among hospital personnel, ${ }^{6}$ prevention of intravascular catheter-related infections, prevention of catheter-associated urinary tract infections, ${ }^{8}$ isolation precautions in hospitals, ${ }^{9}$ prevention of healthcare-associated pneumonia ${ }^{10}$ and prevention of surgical site infections (SSI). ${ }^{11}$ Three of the six interventions included in the Institute for Healthcare Improvement (IHI)'s "100,000 Lives" campaign were collections ("bundles") of guidelines, aimed at reducing three major HAI: ventilator-associated pneumonia (VAP), central-line related bloodstream infections (CRBSI) and SSI. ${ }^{12}$ Guideline compliance, however, is variable, ranging anywhere from $20 \%$ to $100 \% \cdot{ }^{13-15}$ Leading barriers to compliance include unawareness or unfamiliarity, ${ }^{16}$ disagreement over guidelines, ${ }^{17}$ ineffective inter-provider communication, ${ }^{18}$ high workload ${ }^{19}$ and failure of the intensive care unit (ICU) culture to adapt to a new practice. ${ }^{18}$

Consistent compliance with guidelines may be viewed as a systems property ${ }^{20}{ }^{21}$ of ICUs, as well as the result of decisions and habits of individual care providers. Recent studies have revealed that a significant amount of variance in guideline compliance can be explained at the facility level ${ }^{22}{ }^{23}$ in addition to the provider level. ${ }^{24}{ }^{25}$ Therefore, a systems approach is a logical method to understand guideline compliance as a consequence of the interactions between care providers across time, locations, and patients, while simultaneously taking into account the physical and cultural components of an ICU as a system. Although the impact of individual care providers' beliefs and attitudes toward guideline compliance is well studied, the use of a systems approach to understand variance in compliance is very limited. ${ }^{26}$

We conducted a qualitative study in two ICUs to uncover why ICUs as work system ${ }^{21}{ }^{27}$ do not comply consistently with the evidence-based guidelines given in Box 1 using a grounded theory approach. ${ }^{28}$ The grounded theory approach ${ }^{28} 29$ is a qualitative research methodology that emphasises the iterative nature of discovery. It is well suited for research designed to identify major categories 
of behaviour patterns, such as compliance with evidence-based guidelines, using a generative approach as opposed to a confirmative approach. Rather than a preconceived theory or concept in mind, this study was initiated with one broad research question: "Why do ICUs not comply with evidencebased guidelines consistently?" We also attempted to identify the strategies developed and implemented by these ICUs to improve guideline compliance as the characteristics of these strategies provide insight to answer the broad research question.

\section{METHODS}

\section{Setting and sample}

The study was approved by the Institutional Review Board and conducted in two surgical intensive care units (SICU), in two different urban hospitals. SICU-A was a 19-bed unit in a large, university-affiliated teaching hospital with 991 admissions and average length of stay (LOS) of 5.5 days in 2006. SICU-B was a 10-bed unit in a Veterans Affairs Medical Center (VAMC) with 252 admissions and average LOS of 5.8 days for the same time period. A total of 20 care providers in seven roles (attending physicians, residents, nurses, infection control practitioners, quality improvement coordinators, respiratory therapists and pharmacists) were interviewed. Convenience sampling was used to recruit care providers within each role.

\section{Data collection}

Semistructured interviews were conducted with a written interview guide by a single interviewer (APG). A list of the four chosen clinical guideline bundles (box 1) was provided to

Box 1 Evidence-based guidelines to prevent four leading types of healthcare-associated infections

Ventilator-associated pneumonia ${ }^{10} 3031$ :

- elevation of the head of the bed $\left(>30^{\circ}\right)$;

- daily sedation interruption and daily assessment of readiness to extubate;

- peptic ulcer disease prophylaxis;

- daily chlorhexidine oral rinse.

Central venous catheter-related bloodstream infections ${ }^{72}$ :

- hand hygiene;

- maximal barrier precautions;

- optimal catheter site selection, with subclavian vein as the preferred site for non-tunneled catheters;

- chlorhexidine skin antisepsis;

- daily review of line necessity, with prompt removal of unnecessary lines.

Surgical site infections ${ }^{11}{ }^{33}$ :

- discontinuation of prophylactic antibiotics within $24 \mathrm{~h}$ after surgery;

- maintenance of postoperative tight glucose control;

- establishment of postoperative normothermia.

Catheter-associated urinary tract infections ${ }^{8}{ }^{34}$ :

- regular assessment of continued need for catheter;

- sterile technique at insertion;

- daily meatal care;

- drainage bag below the patient's bladder at all times, including during transport;

- anchor catheters to the thigh;

- use of silver-coated catheter in selected cases. the interviewees for reference during interviews. The interview guide was developed, based on meetings and observations in the SICUs. Pilot interviews with five participants were conducted to ensure the clarity of the questions and effectiveness in prompting thoughtful responses and ideas. The interview guide (sample items in box 2) included two general open-ended questions and prompts to explore a particular topic. Clarifications and follow-up questions were used to further understand themes emerging from different interviewees' views and perceptions. Each interview was recorded and transcribed verbatim. Any mentioning of physical artefacts (eg, information sheets, central venous catheter (CVC) insertion cart) in the interviews was followed up with a request for demonstration of the artefacts, as well as questions regarding their use.

\section{Data analysis}

Data were analysed iteratively using a three-step coding process: open coding, axial coding and selective coding. ${ }^{28}$ In the open coding phase, two authors (APG and KLS) examined the data in detail using the constant comparative approach and developed initial categories. The interview transcripts were first segmented and then annotated to identify key themes. A preliminary list of categories was developed, including definitions for each category. In the axial coding phase, categories were systematically developed, refined and linked with subcategories. A definition for each category was developed. At this stage, we conducted two group sessions during which four authors (APG, YX, KLS and VV) interacted and shared ideas to increase insight into the process of refining the categories and relating categories to subcategories. We assessed whether (1) each category and subcategory was clearly defined, (2) there were any overlapping categories and (3) alternative categorisations exist. Categories were revised accordingly and reduced from an initial of 12 to five. In the selective coding phase, a central category, systems ambiguity, emerged as a concept to explain the variation and

\section{Box 2 Sample items from the interview guide}

\section{General items}

Quality and safety in intensive care units can be improved by consistently following the guidelines listed on the information sheet given to you. For each of the guidelines listed, please answer the following questions:

- What are some of the leading problems and barriers encountered in your surgical intensive care unit (SICU) that may hinder compliance with this guideline?

- What are some of the strategies and tools you have implemented to improve compliance with this guideline in your SICU?

Specific items/prompts (sample items)

- Who are the primary care providers responsible for ensuring compliance with this guideline? Can you describe their roles with regards to complying with this guideline?

-What information do you need to be able to follow this guideline?

- How do you know whether daily sedation interruption and daily assessment of readiness to extubate was conducted for a particular patient on a specific day?

- How do you find out when a central venous catheter was inserted?

- What are your practices to reduce central venous catheterrelated bloodstream infections? What are the common lapses in compliance? 
Table 1 Demographic characteristics of the participants (labels are used below in referencing sources of quotes)

\begin{tabular}{|c|c|c|c|}
\hline $\begin{array}{l}\text { Interviewee } \\
\text { no }\end{array}$ & Position & Label & $\begin{array}{l}\text { Experience in the } \\
\text { study site }\end{array}$ \\
\hline \multicolumn{4}{|l|}{ SICU-A } \\
\hline 1 & Attending physician & AA1 & 5 years \\
\hline 2 & Nurse & NA1 & 3 years \\
\hline 3 & Nurse & NA2 & 8 years \\
\hline 4 & Nurse & NA3 & 1 year \\
\hline 5 & Infection control practitioner & IA1 & 2 years \\
\hline 6 & Quality improvement coordinator & QA1 & 2 years \\
\hline 7 & Quality improvement coordinator & QA2 & 4 years \\
\hline 8 & Resident & RA1 & 8 weeks \\
\hline 9 & Respiratory therapist & TA1 & 4 years \\
\hline 10 & Pharmacist & PA1 & 6 years \\
\hline \multicolumn{4}{|l|}{ SICU-B } \\
\hline 11 & Attending physician & $\mathrm{AB} 1$ & 3 years \\
\hline 12 & Attending physician & AB2 & 7 years \\
\hline 13 & Nurse & NB1 & 5 years \\
\hline 14 & Nurse & NB2 & 2 years \\
\hline 15 & Nurse & NB3 & 10 years \\
\hline 16 & Infection control practitioner & IB1 & 5 years \\
\hline 17 & Quality improvement coordinator & QB1 & 6 years \\
\hline 18 & Resident & $\mathrm{RB} 1$ & 4 weeks \\
\hline 19 & Respiratory therapist & TB1 & 7 years \\
\hline 20 & Pharmacist & PB1 & 9 years \\
\hline
\end{tabular}

SICU, surgical intensive care unit.

the main points in the data. We defined systems ambiguity as "uncertainty or vagueness that may prevent the system from achieving its purpose." Finally, the five categories and subcategories were related to the systems ambiguity concept through explanatory statements of relationships.

After completing the coding process as outlined by Strauss and Corbin, ${ }^{28}$ inter-rater reliability was assessed. Two coders (APG and KLS) analysed two randomly chosen interviews (interviewees \#1 and \#11 in table 1, for a total of 27 segments) independently. Among the 27 segments, two were coded differently. The coders discussed the differences in the coding. Other authors contributed to the process by critically reviewing the coded data and making suggestions. Consequently, two descriptions in the categorisation structure were slightly modified to reduce any confusion in the coding process. The final categorisation structure (table 2 ) was agreed by all authors.

\section{RESULTS}

Semistructured interviews were conducted over a 6-month period beginning in April 2006 (table 1). The average duration of the interviews was 27 min (ranges 14-66 min).

Boxes 3-6 provide examples for each type of ambiguity that impede compliance with the guidelines given in box 1 as well as the strategies developed to remove the ambiguity. The ambiguity types are illustrated below briefly, with examples and quotes from interviewees.

\section{Task ambiguity}

To complete the patient care tasks safely and thoroughly as required by guidelines, a care provider needs information on which tasks to complete for which patients, what has already been done for which patient, when to complete these tasks, and the goals for the patient. Lack of clarity in the goal(s) for the patient was reported to hinder compliance with guidelines as noted by an attending physician when asked about the daily sedation interruption for ventilated patients:

A lot of times [night] nurses will keep on giving sedation to patients, especially for preventing patients from moving around and extubating themselves... You have to DC [discontinue] all the orders because if you give PRN orders, patients are going to get it at night. You really have to have a clear goal on what this patient needs and the nurse needs to understand that... If not, you will go to the unit in the morning and find that patient is not awake, and therefore cannot participate in the weaning trial. (AB2; see table 1)

Various strategies were developed by the SICUs to reduce task ambiguity such as providing visual cues, clearly specifying what needs to be done for the patient and standardising processes. For example, in patient populations that are at an increased risk for acquiring VAP, providing visual cues for keeping the head of bed $>30^{\circ}$ by posting notes and reminders in the unit was reported as a strategy used to reduce task ambiguity: "For patients vulnerable to aspiration, like ENT patients, physicians type [head of bed $>30^{\circ}$ ] in the computer as an order. The unit secretary will then actually print this order in large, bold letters and post it in patient's room." (NB3).

Table 2 Ambiguity types in intensive care units leading to inconsistency in complying with guidelines

\begin{tabular}{|c|c|}
\hline Type of ambiguity & Description \\
\hline \multirow[t]{5}{*}{ Task ambiguity } & Patient: Do I know which guidelines are applicable for my patients? \\
\hline & Status: What part of the guideline had already been performed? What part had not? \\
\hline & Plan: What task(s) need to be completed for this patient per guidelines? \\
\hline & Timing: When should this task be completed per guidelines? \\
\hline & Goal: What is the goal for the patient with respect to a particular guideline? \\
\hline \multirow[t]{3}{*}{ Expectation ambiguity } & Standards: What are the acceptable practices in this unit regarding complying with a particular guideline? What is the regular practice? \\
\hline & $\begin{array}{l}\text { Performance: How is my performance regarding complying with guidelines? How is the performance of the unit? Will compliance be part of my } \\
\text { performance evaluation? }\end{array}$ \\
\hline & Feasibility: Is it feasible to follow this guideline in addition to my other responsibilities? \\
\hline \multirow[t]{3}{*}{ Responsibility ambiguity } & Role: Who is responsible for completing this step of the guideline? \\
\hline & Accountability: Which care provider(s) are accountable for the consequences of deviations from a particular guideline? \\
\hline & Authority: Who has the authority to make a decision with regards to applicability of a guideline for a particular patient? \\
\hline \multirow[t]{4}{*}{ Method ambiguity } & Procedural: How to complete a particular step of a guideline? \\
\hline & Source: Where to find the necessary information on a step of a guideline? \\
\hline & Supplies and equipment: Where and how to find the necessary supplies and equipment needed for following a guideline? \\
\hline & Help: Who to contact for help with following a guideline? How to contact? \\
\hline \multirow[t]{2}{*}{ Exception ambiguity } & Should this patient be excluded from this guideline due to her conditions? \\
\hline & What are the exclusionary conditions of this guideline to avoid additional risk to the patient or an affect on the patient's comfort? \\
\hline
\end{tabular}




\section{Ambiguity examples:}

- Goals for ventilator weaning trials are unclear for night shift nurses (task ambiguity): Weaning trials typically are conducted during the first couples of hours of the day shift (07:00 to 09:00), which necessitates a sedation interruption to be started during the night shift preceding the trial. The plan for conducting weaning trials is frequently not clearly communicated to the night shift nurses. As a result, the night shift nurse often focuses on keeping patients comfortable over night and therefore does not reduce sedation. Patients are then still heavily sedated in the morning, which leads to delays in weaning trials (AB1, $A B 2)$.

- Uncertainty about how to conduct the daily sedation interruption (method ambiguity): There is no guideline for how to conduct the daily sedation interruption, so the method of doing it is nebulous among nurses (NA1, QA1, AB2).

- Uncertainty about how to conduct the weaning trial (method ambiguity): There is no weaning trial protocol for respiratory therapists to follow, so the method of conducting a weaning trial varies with each attending physician and ventilator type. Most of the time, the respiratory therapist does not start a weaning trial unless there is an order from physician specifying how to do the trial, which may delay weaning of the patient (AB2, TB1, RB1).

- Uncertainty regarding complying with $\mathrm{HOB}>30^{\circ}$ for particular patient populations (exception ambiguity): Nurses frequently are unsure about complying with $\mathrm{HOB}>30^{\circ}$ for obese patients due to problems with their breathing when their $\mathrm{HOB}$ is kept above $30^{\circ}$ (NB2, TA1, AA1).

\section{Example strategies to reduce ambiguity (type of ambiguity targeted in parentheses):}

- Providing visual cues in patient rooms to consistently keep $\mathrm{HOB}>30^{\circ}$ for patients at high risk for ventilator-associated pneumonia (task ambiguity): For ENT patients, the physician order for $\mathrm{HOB}>30^{\circ}$ is printed in large, bold font by the unit secretary and posted in patient's room (NB3).

- Giving specific orders for daily sedation interruption (task ambiguity): Instead of giving PRN (pro re nata—as needed) orders, physicians write specific orders for sedation over night so that the goal of a daily sedation interruption is clear for the night shift nurse (NB1, AB2).

- Standardised orders (task ambiguity): Peptic ulcer prophylaxis is included as part of the SICU admission order set. Physicians can choose which prophylaxis to order from a prestructured form that becomes part of the patient's admission orders (PA1).

- Increasing awareness about individual and unit performance with ventilator-associated pneumonia prevention (expectation ambiguity): Infection control practitioners, senior nurses, and quality improvement coordinators participate in monthly performance improvement meetings and discuss the ventilator-associated pneumonia rates of the last month. After the meeting, senior nurses relay this information to SICU staff, and give feedback to individual nurses about their performances. Monthly ventilator-associated pneumonia rates are also posted in the unit by infection control (QB1, IA1).

- Clarifying the roles of different care providers in the daily sedation interruption and weaning trials (responsibility ambiguity): The role of each care provider involved in the daily sedation interruption and weaning trial was clarified through in-services. Each role was described in detail, highlighting how the timely and consistent completion of the process depends on each care provider fulfilling their responsibilities in a timely manner (QB1).

\section{Expectation ambiguity}

Individual care providers are more likely to comply with guidelines if they know the norms and expectations of a unit regarding compliance with a particular guideline, are periodically informed about the unit's and their own compliance rates with the guideline and believe in the feasibility of consistently complying with the guideline in addition to their existing workload and responsibilities. Interviewees in both SICUs were poorly informed about the expectation and performance in complying with the four guidelines. One nurse stated: "The monthly infection rates are written somewhere in the unit in a folder, but nobody spends time to find and look at them... I have no idea what our infection rates were last month." (NB1)

Participating SICUs reported using several training methods to reduce expectation ambiguity, such as distributing hand-outs highlighting key points regarding guidelines, short training sessions during staff meetings, and one-on-one training. Oneon-one training includes quality improvement nurses talking with each nurse in the unit individually to emphasise the importance of following particular guidelines:

Talking face to face had an impact because instead of in-servicing like sitting through the motion and listening, having an individual, one to one conversation had probably worked with the VAP because we were able to not only explain the importance of following guidelines and increase their awareness, but also get feedback from the nurses why [compliance with guidelines to prevent VAP] is not happening. (OB1)
An auditing mechanism that tracked individual nurses' performance was used to clarify and reinforce expectations when introducing new guidelines:

[Quality improvement nurses] go around to rooms and look to see if nurses are following the insulin protocol properly. According to the new protocol, people should be following the q1 fingersticks until the blood sugar is less than 120. Our old protocol was q2 h, so everyone is kind of in that habit... We go around individually and check the charts and if the nurses are out of range, we speak with them and let them know that this is what they need to be doing. (OA2)

\section{Responsibility ambiguity}

Guideline compliance can be improved by clarifying who is responsible for a specific task, who has the authority to make a decision in regards to applicability of a guideline for a particular patient, and who will be accountable for compliance with a particular guideline. An example of responsibility ambiguity is related to the tight glucose control (TGC) guideline, which is a shared responsibility between nurses and physicians. It was reported that once an order for TGC was written, some physicians thought that it was the nurses' responsibility to get and keep the glucose under control and thus did not pay attention to the out-of-range glucose levels: 
Ambiguity examples:

- Lack of information about time of central venous catheter insertion (task ambiguity): If a central venous catheter was inserted before the patient was admitted to SICU, information about when the line was inserted usually is not known to SICU staff (NA3, RA1).

- Lack of clarity about the location of supplies necessary for central venous catheter insertion (method ambiguity): Different supplies used in central venous catheter insertion are stocked in different areas of the stock area. Physicians have to search for supplies in the stock area and bring them together before each insertion (NB3, RB1).

- Unclear feedback given regarding central venous catheter-related bloodstream infections (expectation ambiguity): Presenting bloodstream infection rates in the format of 4.6 per 1000 line days is not perceived by bedside nurses as directly relevant to their practice (QA1).

Example strategies to reduce ambiguity (type of ambiguity targeted in parentheses):

- Use of information tools that provide an overview of key information related to central venous catheters of patients in the unit (task ambiguity): A one-page paper-based form that includes information on the status of each patient's central venous catheter(s), including the times of the line insertions and dressing changes, was created. The form is used during multidisciplinary rounds for reviewing line necessity, and is used during handoffs to make sure that information necessary for line management is transferred effectively. The form also facilitates cross-monitoring (NB1, AB1, IA1).

- Reduce ambiguity by providing information to connect individual care providers' activities to specific occurrences of central venous catheter-related bloodstream infections (expectation ambiguity): Feedback on performance is given to all care providers involved in the care of a patient immediately after a central venous catheter-related bloodstream infection is discovered. Care providers who inserted the central venous catheter, who changed dressing, etc all meet and hear about this case rather than only managers, infection control and performance improvement coordinators (IA1, QA1).

- Clear role assignment (responsibility ambiguity): One resident is assigned to remove or change central venous catheters every day based on decisions made during multidisciplinary rounds (RA1).

- Putting all supplies necessary for central venous catheter insertion together (method ambiguity): A cart including all supplies necessary for inserting a central venous catheter was developed. The cart is periodically restocked. Having all of the necessary supplies in one location facilitated compliance with central venous catheter insertion guidelines, such as large drapes for maximum barrier precautions (QA1, RA1, IA1).

- Training to new residents on guidelines to prevent central venous catheter-related bloodstream infections (expectation ambiguity): All new residents rotating through the unit are provided training on central venous catheter insertion standards and expectations before inserting any catheters (AB1, IB1).

\begin{abstract}
During morning rounds, the resident may report the glucose range from 140 to 160 and no one in the team says anything. It is nurses' responsibility to keep it that tight but if it is not that tight no one follows up with it. No one asks why is it above 120 ? Why can't we get it under control? Then, the nurses will think "Well, they did not mention anything so what I am doing is probably fine." (OA1)
\end{abstract}

One of the quality improvement coordinators (OA1) thought that ideally both the nurse and the physician should share the responsibility of keeping the glucose level in the accepted range. If, for some reason, the nurse was unable to achieve TGC, the physician should have worked with the nurse to understand the reason and find a solution. In one SICU, nurses were primarily responsible for performing hourly glucose checks and administering insulin to keep the glucose level in the defined range, and physicians were responsible for checking patients' glucose levels periodically and finding a solution if the glucose level could not be controlled in spite of the intervention.

The interviewees reported ambiguity in linking an individual's compliance with guidelines to infectious outcomes of care. They cited examples of care providers not washing hands before touching the patient and residents not following maximal barrier precautions, but they did not associate the non-compliance with guidelines to the development of HAIs in their patient populations. One strategy reported to reduce responsibility ambiguity was to provide feedback to all care providers involved in the care of a specific patient immediately after infection was detected:

We get together and say that we had a BSI with Mr [Jones]. The person

who inserted the line is there [in the meeting], the nurse is there,

everyone involved in the care of this patient is there... with real-time reporting people feel like "I was involved in the care of this patient, I changed the dressing on this patient, so I am accountable. (RB1)

\section{Method ambiguity}

Method ambiguity was reported to occur due to the combined effect of guideline complexity and the demanding ICU work environment. For example, one nurse said:

\footnotetext{
The tight glucose protocol is very wordy and I don't have enough time to go and look through it...l've developed my own way of calculating the insulin that needs to be given. I've done this job quite enough that I can guess how much insulin to give based on trends [in patient's condition]... I feel like it's [following the TGC] going to take more time to achieve the goal than I've been able to achieve already [using my own method]. (NA1)
}

Being able to quickly find the necessary supplies without spending time searching was reported as a key factor in complying with some guidelines. For example, the two periodically restocked CVC insertion carts in SICU-A included all the supplies necessary for inserting the catheters. One resident noted: "It is really easy to comply with the CVC insertion guidelines in this unit because everything you need is available on the cart." (RA1)

\section{Exception ambiguity}

Interviewees reported ambiguity in how to decide exceptions to guidelines when the patient's condition warranted exceptions, such as when the potential risks or perceived patient discomfort outweighed the benefits of the guideline. Nurses reported ambiguity in balancing guideline benefits with potential to 
Box 5 Examples of different types of ambiguity that hinder compliance with guidelines to prevent surgical site infections and strategies to remove ambiguity

Ambiguity examples:

- Lack of clarity about the timing of prophylactic antibiotics administration for postop patients (task ambiguity): Due to the inefficiency in the information systems, it is often hard for nurses to find the information about when the prophylactic antibiotics were first administered preoperatively, which complicates the timing of subsequent administrations in SICU (AB1, PA1).

- Lack of common understanding that maintaining glucose level of patients under control is a shared responsibility between nurses and physicians (responsibility ambiguity): Both nurses and physicians should share the responsibility of keeping a patient's glucose level in the accepted range, but there is ambiguity regarding this shared responsibility and the roles of different care providers in the process. For example, if a patient is put on the tight glucose control protocol, but the glucose level still cannot be kept under control, some physicians may think it is nurses' responsibility to get the glucose level under control and do not pay attention to the out-of-range values. When physicians do not mention anything, the nurse thinks what she is doing must be fine (OA1).

- Uncertainty about how to maintain glucose level in the acceptable range (80-120 mg/dl) (method ambiguity): Following tight glucose control guideline for calculating how much insulin to titrate on an hourly basis is hard due to the complexity and wordiness of the guideline. Nurses cannot find adequate time to go through the complex algorithms. This becomes even more of a problem if a nurse has two patients on the tight glucose control guideline on the same shift. Instead of using the lengthy and complex guideline, nurses will often titrate the insulin based on their own judgements and experiences (NA1, AA1).

Example strategies to reduce ambiguity (type of ambiguity targeted in parentheses):

- Standardised orders (task ambiguity): Tight glucose control is included as part of the standard SICU admission orders for all patients except patients with a pancreas transplant (NA2, NA3, PA1).

- Regular feedback to each individual nurse about compliance with tight glucose control guidelines (expectation ambiguity): Intensive auditing is conducted, and detailed feedback is provided to each nurse periodically about their performance in complying with the hourly finger stick glucose level checks (QA2, NA3).

significantly compromise patient's comfort and sleep. They also reported their fear of putting their patients at higher risk when following guidelines. One quote illustrates such concerns:

\section{It is not typical in the SICUs that some of the things known to prevent UTI such as the leg straps are used. Patients have oedema and it [leg strap] gets too tight around their legs and may restrict blood flow. Then, you start to ask: Does this apply to this patient or does it not? (OA1)}

Daily sedation interruption and a subsequent breathing trial was one guideline that care providers in both SICUs reported deviating from on occasion due to the patient's condition:

I look at the patient rather than what the guideline says... You have a patient that is very wild or nasally intubated, you reduce the sedation and they wake up, sit up and take the tube out, that's more of a problem for me than waiting maybe for another day. I try to look at my other parameters and go from there. I don't want to jeopardise someone's lines or their tubes. If I can wean their sedation, of course I will. But to me it's a patient safety issue... Physicians order sedation vacation and weaning trials. We may agree or disagree with the order. (RA1)

\section{DISCUSSION}

Our qualitative study identified some of the underlying causes of non-compliance with evidence-based guidelines directed at prevention of the four major HAIs. The concept of systems ambiguity emerged as a source of non-compliance with guidelines. Five types of ambiguity (task, expectation, responsibility, method and exception) presented in table 2 provide useful insight into the root causes of non-compliance, thus giving hints to possible strategies for improving compliance with guidelines. Our findings suggest that much ambiguity exists in the ICU setting, and most likely in other healthcare settings, which hinders consistent compliance with guidelines. A direct implication of these findings is that organisations should explore different ways to reduce ambiguity, and as a result increase the likelihood of compliance with guidelines.

Our findings on HAIs using the concept of systems ambiguity support the relevance and significance of the concept of ambiguity for the patient safety literature. Spear and Schmidhofer ${ }^{35}$ recently argued that ambiguity in healthcare organisations contributes to medical errors and is an obstacle for organisational learning and continuous process improvement. The authors further suggest that healthcare organisations should aim to reduce ambiguity by clarifying the answers to the following questions: (1) What is the goal of the healthcare organisation? (2) Who is responsible for what tasks? (3) How is information, materials, or services exchanged? and (4) How are tasks performed? ${ }^{35}$ For example, a qualitative study conducted in the operating rooms of two hospitals suggested that compliance with prophylactic antibiotic administration can be improved by reducing ambiguity regarding several issues ${ }^{36}$ : clearly identifying the patients who need antibiotics, the type of antibiotics that should be administered, the care provider

Table 3 Comparison of the "barriers to guideline adherence framework" developed by Cabana et $a l^{16}$ and the systems ambiguity concept

Four elements of the "barriers to guideline adherence framework" by Cabana et al ${ }^{16}$ Lack of agreement with a particular guideline - applicability to a particular patient Lack of agreement with guidelines in general—guidelines are not practical Patient preferences - inability to reconcile patient preferences with guideline recommendations

Guideline characteristics-not easy to use, not convenient, cumbersome, confusing
Ambiguity types from the systems ambiguity concept presented in table 2

Exception ambiguity

Expectation ambiguity

Exception ambiguity

Task ambiguity

Method ambiguity 
Box 6 Examples of different types of ambiguity that hinder compliance with guidelines to prevent catheter-associated urinary tract infections and strategies to remove ambiguity

Ambiguity examples:

- Lack of clarity about when a foley catheter was inserted (task ambiguity): If a foley catheter was inserted before admission to the unit or the hospital, the SICU staff may not know when it was inserted, which ultimately may affect the decision regarding when to remove it (OA1, AB2).

- No effective feedback mechanism on unit performance regarding catheter-associated urinary tract infections (expectation ambiguity): Monthly catheter-associated urinary tract infection rates for SICU are kept in a file on the unit, but there is no mechanism for enforcing that staff reviews these rates (RA1, NB2).

- Uncertainty about the applicability of the guideline that requires securing the foley catheter to the leg (exception ambiguity): Nurses question the applicability of using leg straps to secure foley catheters in patients with significant oedema. In this patient group, the leg strap may be too tight around the leg and restrict blood flow (QA1).

Example strategies to reduce ambiguity (type of ambiguity targeted in parentheses):

- Training nurses and patient care technicians on the importance of avoiding cross contamination by using separate drainage containers for each drainage type (expectation ambiguity): A majority of the nurses and patient care technicians think that it is acceptable to empty all the drainage from one patient into a single container before discarding it. However, the nozzle of the foley bag may hit the rim of this single container and cause cross-contamination, which may lead to urinary tract infection. A short training session for nurses and patient care technicians was created to clarify the rationale for having dedicated containers for each drainage type (OA2, IA1).

responsible for administering the antibiotics, and clearly setting the goal of administering the antibiotics before the patient is brought to the operating room.

Many of the factors that affect compliance with evidencebased guidelines identified in the literature may be explained by the concept of systems ambiguity. For example, based on a review of 76 studies for identifying barriers to physicians' adherence to guidelines, Cabana et a ${ }^{16}$ developed a conceptual model of "barriers to guideline adherence." As can be seen from table 3 , four of the barriers in this model can be explained by at least one of the five "system ambiguities" identified in the present study (table 2). In addition, we used the systems ambiguity concept to identify several other factors such as ambiguity in status, timing, goal and accountability that were not identified by Cabana et al. ${ }^{16}$ Recently, Hysong et al ${ }^{26}$ found that hospitals with care providers that have a clear and focused shared mental model of evidence-based guidelines and a good feedback mechanism that promotes learning from mistakes and successes have better patient outcomes. Having a clear, shared mental model can be achieved through reducing ambiguity in the system and providing effective feedback (a method to reduce expectation ambiguity) is one way to achieve that.

Although the systems ambiguity concept provides an explanation for many causes of non-compliance with evidence-based guidelines, factors besides systems ambiguity also play an important role. Some examples of factors not included in our systems ambiguity concept that have been previously identified in the literature include high workload ${ }^{19}$ and understaffing, ${ }^{37}$ forgetfulness, ${ }^{19}{ }^{38}$ disagreement with the guideline, ${ }^{39}$ perception of significant increase in costs with guideline implementation, ${ }^{40}$ and lack of necessary supplies and equipment. ${ }^{39}{ }^{41}$ Staff attitude toward guidelines is an important predictor of guideline compliance and has been shown to vary by profession ${ }^{42}{ }^{43}$ and type of healthcare setting. ${ }^{24} \mathrm{~A}$ recent survey study conducted in 70 US ICUs revealed that staff in paediatric ICUs have significantly more positive attitudes towards evidence-based guidelines than the staff in adult ICUs. ${ }^{24}$ Furthermore, nurses and other staff had more positive attitudes towards guidelines than physicians. ${ }^{24}$ In summary, the systems ambiguity concept expands and complements the existing literature on factors affecting compliance with evidence-based guidelines, but it is does not explain all the causes of non-compliance behaviours.

Besides providing an explanation for non-compliance, we believe the concept of systems ambiguity also provides an insightful way to identify intervention strategies to improve guideline compliance. This is supported by published reports of the successful interventions to improve compliance with guidelines and reduce HAI (table 4). Several of the strategies identified in our study to reduce ambiguity (boxes 3-6) have also been reported in the literature as successful interventions to improve compliance with the guidelines and reduce HAIs (table 4): training ${ }^{44-46}$ and providing feedback, ${ }^{47-49}$ computerised decision-support tools, ${ }^{50}$ standardisation of processes ${ }^{46}$ and use of checklists. ${ }^{45}$ Our study identified several other interventions not reported in the literature, such as designing information tools that provide an overview of key information required for guideline compliance, having the physicians being more specific with orders rather than PRN orders whenever possible, and clarifying the roles of each care provider in carrying out the steps required for each guideline.

Although our findings suggest reducing systems ambiguity to improve guideline compliance and decrease HAIs, guidelines should not replace the critical and educated judgement of care providers. There will always be some ambiguity with regard to guideline compliance due to the uniqueness of each patient. In addition, overcompliance or unquestioning compliance can have unintended and serious consequences that can harm patients. We believe that the importance of our findings lies on the systems approach and on viewing and studying ICUs as work systems. ${ }^{21}$ When such work systems have less ambiguity, care providers will be more likely to comply with guidelines.

As our study focused on compliance with best practice guidelines directed at the reduction of HAIs, findings have implications for infection control practice. For each type of ambiguity, we have summarised strategies that may provide clarity to the guidelines and promote consistent compliance.

\section{Reducing task ambiguity}

During the provision of care, it must be clear to care providers which guidelines are applicable for a particular patient, what tasks have been completed and which tasks need to be completed, when a particular task should be completed and 
Table 4 Interventions reported in the literature to improve guideline compliance and the corresponding ambiguity type targeted

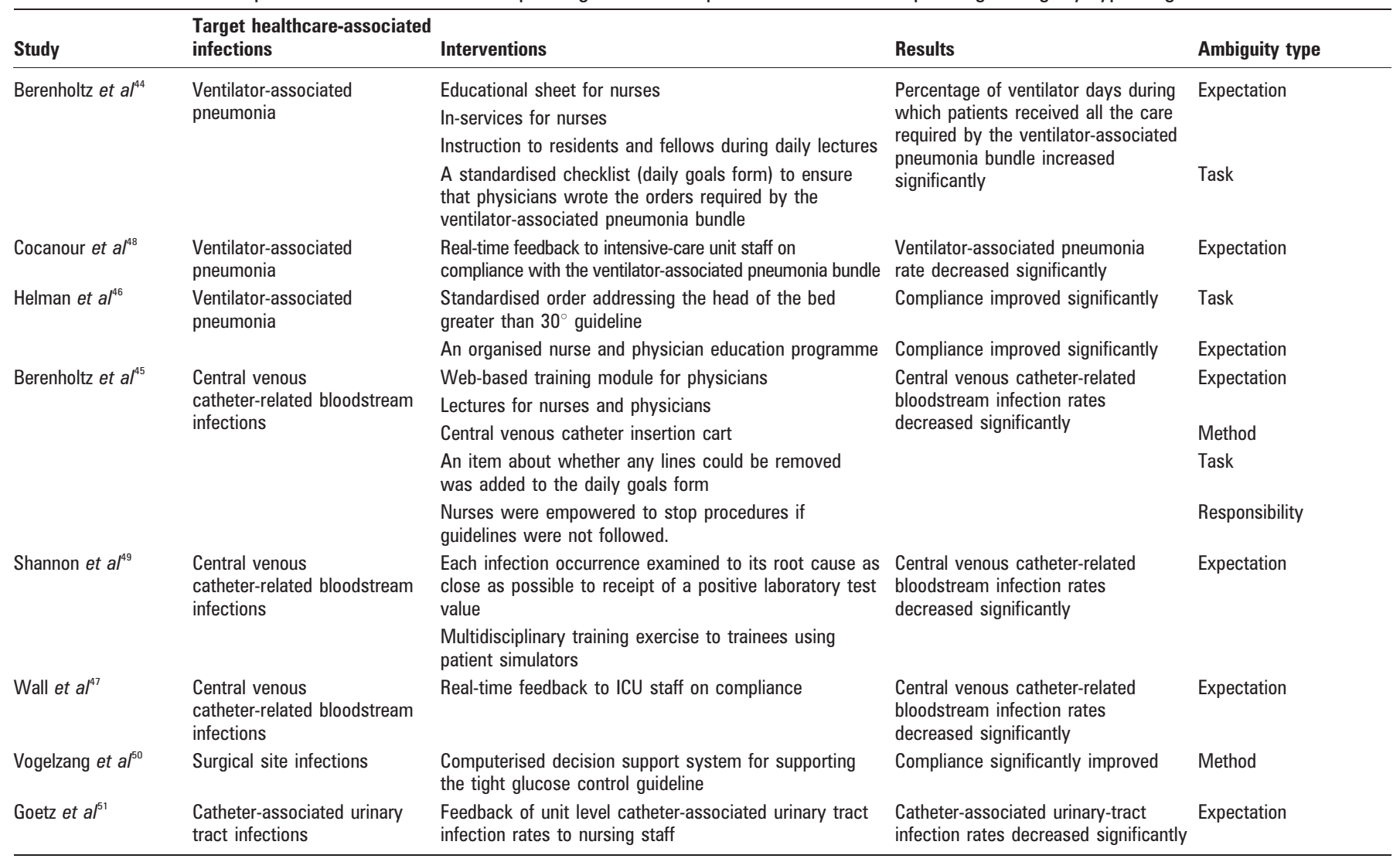

ICU, intensive care unit.

the goal for each patient with respect to a particular guideline. We advocate for the design and implementation of information technology solutions to address these issues. Information technology can remove ambiguity by providing the most relevant information necessary for guideline compliance in the time-constrained care settings. Since compliance with guidelines frequently relies on removing ambiguity associated with coordinating activities between care providers, process-oriented information tools ${ }^{52}$ that are invaluable in coordination of care, such as the notes posted in patient rooms to keep the $\mathrm{HOB}$ $>30^{\circ}$ (box 3) and the one-page paper-based form providing information on the status of CVCs for each patient in the unit (box 4), may be effective.

\section{Reducing expectation ambiguity}

Each care provider in a healthcare setting should have a clear understanding of what is expected of them in terms of complying with guidelines and preventing HAIs. This understanding should include how compliance with guidelines will be assessed on an individual level (eg, performance evaluation) as well as a unit-based level (eg, meeting the infection reduction targets established for the unit). New, innovative, and more participative training methods are needed to supplement the traditional didactic training approaches to infection control education in order to reduce expectation ambiguity. Future research in the area of infection control is needed to understand how to reinforce consistent compliance with guidelines and provide effective performance feedback that leads to individual and organisational learning. In addition, we need to address the challenge of holding care providers responsible and accountable for guideline compliance without turning care settings into "blame-culture" environments.
Reducing responsibility ambiguity and exception ambiguity Infection control professionals must work collaboratively with front-line care providers to ensure that the roles and responsibilities of care providers related to compliance with guidelines are well defined. However, clarification as to who has authority to deviate from an aspect of the guideline and holding care providers accountable for non-compliance with guidelines will need to come from the supervisory physician and nursing staff of the care setting. Decision-support tools should be developed to facilitate decisions regarding deviations from a particular guideline.

\section{Reducing method ambiguity}

Compliance with guidelines is enhanced when the necessary supplies and equipment are available and easily accessible to care providers (eg, central line insertion cart). Care providers should be able to access an electronic or manual copy of the guidelines for quick reference. Additionally, the infection control professionals in the organisation should be available to provide consultation and assistance when needed.

Use of the systems ambiguity concept both proactively and retroactively The systems ambiguity concept including the five types of ambiguity (table 2) can be used both proactively and retroactively by infection control professionals. Proactively, it can be used to identify barriers to compliance with evidence-based guidelines directed at HAI reduction before an adverse event occurs. Infection control programmes can utilise this concept prior to the implementation of a new guideline to assess potential risks, similar to the failure mode and effects analysis (FMEA).$^{53}$ Retroactively, it can be used as a diagnostic tool to understand the underlying causes of non-compliance with guidelines, hence to gain insight into and develop interventions to improve compliance. 
This study has several limitations. The sample size was small and represented a convenience sample. It is possible that if more care providers had been interviewed, more themes related to noncompliance may have emerged. The use of a single data-collection method, the semistructured interview, might have biased the results. In particular, care providers might have viewed some of the problems and barriers encountered as "normal," rather than system problems that need to be solved. Another limitation of the study was that both SICUs were in teaching hospitals that probably require educating and setting up the norms and expectations for new coming trainees more frequently. In a non-teaching hospital, the norms and expectations regarding guideline compliance may be communicated to care providers and enforced in a different manner. Future research should use different data collection techniques, such as observations and surveys and should be conducted in a variety of institutions.

In summary, this qualitative study utilised and further developed the concept of systems ambiguity to characterise compliance with evidence-based guidelines directed toward the reduction of HAIs in two SICUs. Five types of ambiguity emerged from our analysis, which may be used to prevent and diagnose system problems in healthcare settings. We encourage researchers to use the concept of ambiguity and the types of ambiguity (table 2) to study systemlevel problems, both proactively and reactively.

Acknowledgements: We would like to thank the care providers of the two participating ICUs for their time and support of the study. We also would like to thank three anonymous reviewers, the editor and Dr R Kane for their constructive comments and helpful suggestions that have significantly improved the quality and presentation of the paper.

Funding: This work was funded by the National Science Foundation (IIS-0534646). The opinions are of those authors and do not necessarily reflect the sponsor's official position.

Competing interests: None declared.

Ethics approval: Ethics approval was obtained.

\section{REFERENCES}

1. Institute of Medicine. Clinical practice guidelines. Washington, DC: National Academy Press, 1990

2. Chassin MR. Practice guidelines: best hope for quality improvement in the 1990s. J Occup Med 1990;32:1199-206.

3. Burke JP. Infection control — a problem for patient safety. N Eng/ J Med 2003;348:651-6.

4. Eggimann P, Pittet D. Infection control in the ICU. Chest 2001;120:2059-93.

5. Boyce JM, Pittet D. Guideline for hand hygiene in health-care settings: recommendations of the Healthcare Infection Control Practices Advisory Committee and the HICPAC/SHEA/APIC/IDSA Hand Hygiene Task Force. Infect Control Hosp Epidemiol 2002;23(Suppl):3-40S

6. Bolyard EA, Tablan OC, Williams WW, et al. Guideline for infection control in healthcare personnel, 1998. Hospital Infection Control Practices Advisory Committee. Infect Control Hosp Epidemiol 1998;19:407-63.

7. O'Grady NP, Alexander M, Dellinger EP, et al. Guidelines for the prevention of intravascular catheter-related infections. Centers for Disease Control and Prevention. MMWR Recomm Rep 2002;51:1-29.

8. Wong ES. Guideline for prevention of catheter-associated urinary tract infections. Am J Infect Control 1983;11:28-36.

9. Garner JS. Guideline for isolation precautions in hospitals. The Hospital Infection Control Practices Advisory Committee. Infect Control Hosp Epidemiol 1996;17:53-80.

10. Tablan OC, Anderson LJ, Besser R, et al. Guidelines for preventing health-careassociated pneumonia, 2003: recommendations of CDC and the Healthcare Infection Control Practices Advisory Committee. MMWR Recomm Rep 2004;53:1-36.

11. Mangram AJ, Horan TC, Pearson ML, et al. Guideline for prevention of surgical site infection, 1999. Hospital Infection Control Practices Advisory Committee. Infect Control Hosp Epidemiol 1999;20:250-78.

12. Berwick DM, Calkins DR, McCannon CJ, et al. The 100,000 lives campaign: setting a goal and a deadline for improving health care quality. JAMA 2006;295:324-7.

13. Halm EA, Atlas SJ, Borowsky LH, et al. Understanding physician adherence with a pneumonia practice guideline: effects of patient, system, and physician factors. Arch Intern Med 2000;160:98-104.

14. Lomas J, Haynes RB. A taxonomy and critical review of tested strategies for the application of clinical practice recommendations: from "official" to "individual" clinical policy. Am J Prev Med 1988;4(Suppl):77-94S.

15. Grimshaw JM, Russell IT. Effect of clinical guidelines on medical practice: a systematic review of rigorous evaluations. Lancet 1993;342:1317-22.

16. Cabana MD, Rand CS, Powe NR, et al. Why don't physicians follow clinical practice guidelines? A framework for improvement. JAMA 1999;282:1458-65.
17. Mottur-Pilson C, Snow V, Bartlett K. Physician explanations for failing to comply with "best practices." Eff Clin Pract 2001;4:207-13.

18. Anger KE, Szumita PM. Barriers to glucose control in the intensive care unit. Pharmacotherapy 2006:26:214-28.

19. Pittet D, Mourouga P, Perneger TV. Compliance with handwashing in a teaching hospital. Infection Control Program. Ann Intern Med 1999;130:126-30.

20. Bogner MS. Error reduction as a systems problem. In: Bogner MS, ed. Human error in medicine. Hillsdale, NJ: Erlbaum, 1994:67-92.

21. Carayon P, Schoofs Hundt A, Karsh BT, et al. Work system design for patient safety: the SEIPS model. Qual Saf Health Care 2006;15(Suppl 1):i50-i8S.

22. Krein SL, Hofer TP, Kerr EA, et al. Whom should we profile? Examining diabetes care practice variation among primary care providers, provider groups, and health care facilities. Health Serv Res 2002;37:1159-80.

23. Davies M, Spears W, Pugh JA. What VA providers really think about clinical practice guidelines. Fed Pract 2003;21:15-30.

24. Quiros D, Lin S, Larson EL. Attitudes toward practice guidelines among ICU personnel: A cross-sectional anonymous survey. Heart Lung 2007:36:287-97.

25. Whitby M, Pessoa-Silva CL, McLaws ML, et al. Behavioural considerations for hand hygiene practices: the basic building blocks. J Hosp Infect 2007:65:1-8.

26. Hysong SJ, Best RG, Pugh JA, et al. Not of one mind: mental models of clinical practice guidelines in the Veterans Health Administration. Health Serv Res 2005:40:829-47.

27. Gurses AP, Carayon P. Performance obstacles of intensive care nurses. Nurs Res 2007:56:185-94.

28. Strauss A, Corbin J. Basics of qualitative research: techniques and procedures for developing grounded theory. Newbury Park, CA: Sage, 1998.

29. Glaser BG, Strauss AL. The discovery of grounded theory: strategies for qualitative research. Chicago: Aldine, 1967.

30. DeRiso AJ, Ladowski JS, Dillon TA, et al. Chlorhexidine gluconate $0.12 \%$ oral rinse reduces the incidence of total nosocomial respiratory infection and nonprophylactic systemic antibiotic use in patients undergoing heart surgery. Chest 1996;109:1556-61.

31. Kollef MH. Prevention of hospital-associated pneumonia and ventilator-associated pneumonia. Crit Care Med 2004;32:1396-405.

32. Mermel LA. Prevention of intravascular catheter-related infections. Ann Intern Med 2000; 132:391-402.

33. van den Berghe G, Wouters $P$, Weekers $F$, et al. Intensive insulin therapy in the critically ill patients. N Engl J Med 2001;345:1359-67.

34. Leone $\mathbf{M}$, Garnier F, Avidan M, et al. Catheter-associated urinary tract infections in intensive care units. Microbes Infect 2004;6:1026-32.

35. Spear SJ, Schmidhofer M. Ambiguity and workarounds as contributors to medical error. Ann Intern Med 2005;142:627-30.

36. Tan JA, Naik VN, Lingard L. Exploring obstacles to proper timing of prophylactic antibiotics for surgical site infections. Qual Saf Health Care 2006;15:32-8.

37. Pittet D, Simon A, Hugonnet S, et al. Hand hygiene among physicians: performance, beliefs, and perceptions. Ann Intern Med 2004;141:1-8.

38. Patarakul K, Tan-Khum A, Kanha S, et al. Cross-sectional survey of hand-hygiene compliance and attitudes of health care workers and visitors in the intensive care units at King Chulalongkorn Memorial Hospital. J Med Assoc Thai 2005;88(Suppl 4):287-93S

39. Cook DJ, Meade MO, Hand LE, et al. Toward understanding evidence uptake: semirecumbency for pneumonia prevention. Crit Care Med 2002;30:1472-7.

40. Tunis SR, Hayward RS, Wilson MC, et al. Internists' attitudes about clinical practice guidelines. Ann Intern Med 1994:120:956-63.

41. Rello J, Lorente C, Bodi M, et al. Why do physicians not follow evidence-based guidelines for preventing ventilator-associated pneumonia?: a survey based on the opinions of an international panel of intensivists. Chest 2002;122:656-61.

42. Stein AD, Makarawo TP, Ahmad MF. A survey of doctors' and nurses' knowledge attitudes and compliance with infection control guidelines in Birmingham teaching hospitals. J Hosp Infect 2003;54:68-73.

43. Ricart M, Lorente C, Diaz E, et al. Nursing adherence with evidence-based guidelines for preventing ventilator-associated pneumonia. Crit Care Med 2003;31:2693-6.

44. Berenholtz SM, Milanovich S, Faircloth A, et al. Improving care for the ventilated patient. Jt Comm J Oual Saf 2004;30:195-204.

45. Berenholtz SM, Pronovost PJ, Lipsett PA, et al. Eliminating catheter-related bloodstream infections in the intensive care unit. Crit Care Med 2004;32:2014-20.

46. Helman DL Jr, Sherner JH III, Fitzpatrick TM, et al. Effect of standardized orders and provider education on head-of-bed positioning in mechanically ventilated patients. Crit Care Med 2003:31:2285-90.

47. Wall RJ, Ely EW, Elasy TA, et al. Using real time process measurements to reduce catheter related bloodstream infections in the intensive care unit. Qual Saf Health Care 2005:14:295-302.

48. Cocanour CS, Peninger M, Domonoske BD, et al. Decreasing ventilator-associated pneumonia in a trauma ICU. J Trauma 2006;61:122-9.

49. Shannon RP, Frndak D, Grunden N, et al. Using real-time problem solving to eliminate central line infections. Joint Commiss J Qual Patient Saf 2006;32:479-87.

50. Vogelzang M, Zijlstra F, Nijsten MWN. Design and implementation of GRIP: a computerized glucose control system at a surgical intensive care unit. BMC Med Inform Decis Mak 2005;5:38

51. Goetz AM, Kedzuf $S$, Wagener $M$, et al. Feedback to nursing staff as an intervention to reduce catheter-associated urinary tract infections. Am J Infect Control 1999;27:402-4.

52. Gurses AP, Xiao Y. A systematic review of the literature on multidisciplinary rounds to design information technology. JAMIA 2006:13:267-76.

53. Stamatis DH. Failure mode and effects analysis: FMEA from theory to execution. Milwaukee, Wl: ASOC Ouality Press, 1995 